\title{
Preparados para la guerra. La construcción de la identidad rusa post-soviética en los discursos de la Victoria
}

\author{
Frederic GUERRERO-SOLÉ \\ Universitat Pompeu Fabra \\ frederic.guerrero@upf.edu. \\ Hibai LÓPEZ GONZÁlEZ \\ Universitat Pompeu Fabra \\ hibai.lopez@upf.edu
}

Recibido: 9 de mayo de 2011

Aceptado: 21 de febrero de 2012

\section{Resumen}

20 años después de la disolución de la Unión Soviética, la celebración de la Victoria sobre la Alemania nazi cada 9 de mayo se ha erigido en Rusia como la principal fiesta nacional y la base de la construcción de la identidad post-soviética, sobre todo a partir del primer mandato presidencial de Vladímir Putin. El acto central de la celebración es el desfile de la Victoria y la lectura del discurso presidencial en la Plaza Roja de Moscú. Este artículo analiza la construcción del ciudadano ruso post-soviètico a través de los once últimos discursos de la Victoria (2000-2010) utilizando como metodología la semiótica narrativa.

Palabras clave: Rusia, post-soviético, discurso, narrativa, identidad nacional

\section{Ready for war.}

Constructing the Russian post-Soviet identity in Victory speeches

\begin{abstract}
20 years after the dissolution of the Soviet Union, the Victory commemoration in Russia has emerged as the main national holiday and the basis for the construction of post-Soviet Russian identity, especially since the first presidency of Vladimir Putin. The cornerstone of the celebration is the Victory parade and the reading of the presidential speech, every 9th of May in the moscovite Red Square. This article discusses the construction of post-Soviet Russian citizen over the last eleven Victory speeches (2000-2010), by means of narrative semiotics as methodological framework.
\end{abstract}

Keywords: Russia, post-Soviet, discourse, narrative, national identity

Referencia normalizada: GUERRERO-SOLÉ, Frederic y LÓPEZ GONZÁLEZ, Hibai (2012): “Preparados para la guerra. La construcción de la identidad rusa post-soviética en los discursos de la Victoria“. Estudios sobre el mensaje periodístico, vol. 18, núm. 2 (julio-diciembre), págs.: 513-529. Madrid, Servicio de Publicaciones de la Universidad Complutense.

Sumario: 1. Introducción. 2. Objetivos 3. Metodología; 3.1. Modelo Actancial. 4. Análisis de los discursos presidenciales; 4.1. Las competencias de los actantes; 4.2. El programa narrativo de enlace. La memoria y el paso del tiempo. 5. Discusión. 6. Conclusión. 7. Referencias bibliográficas.

\section{Introducción}

La disolución de la Unión Soviética en diciembre de 1991 representó el final de la construcción de la utopía comunista y del homo sovieticus. Rusia, como el resto de las repúblicas que formaban parte de la URSS, vivió un periodo turbulento de repentinos cambios políticos, sociales, económicos, ideológicos y culturales, una transición que se extiende hasta nuestros días. De la dictadura del proletariado se pasó a particulares 
versiones de democracia al estilo occidental; del socialismo, el marxismo-leninismo y la protección del Estado a un sistema gobernado por las leyes del liberalismo, y de un ciudadano fiel a las tesis del partido y de la ideología soviética a un ciudadano que tenía en la patria la principal base de su construcción identitaria (Cfr. CASTELLS, 2009). El nacionalismo fue, precisamente, el principal remedio a la amenaza para la conservación de la unidad del pueblo ruso que había significado el desmoronamiento de las bases sobre las que se había construido la identidad del pueblo soviético.

Durante la época comunista, los grandes acontecimientos -ligados a la Revolución, el Trabajo y la Victoria en la II Guerra Mundial (Gran Guerra Patria para los rusos)se habían convertido en uno de los principales instrumentos de difusión y consolidación de la identidad soviética. A ello se le unía la planificación temática que afectaba a todas las formas de producción cultural, y, en particular, a los medios de comunicación electrónicos ${ }^{1}$. La caída del sistema comunista se llevó consigo todas las celebraciones que albergaban alguna relación con el antiguo (y denostado) sistema. No obstante, el Estado necesitaba de algún apoyo para la consolidación de una base ideológica e identitaria que permitiera construir el futuro de Rusia más allá de la nostalgia soviética. De las tres tradiciones sagradas mencionadas anteriormente -la Revolución, el Trabajo y la Victoria (Cfr. LANE, 1981)- sólo esta última cumplía los requisitos necesarios para convertirse en la gran celebración del pueblo ruso y, además, permitía la conservación de la tradición patriótico-militar y de la representación de un poder fuerte, profundamente arraigados en el imaginario colectivo ruso.

Rusia celebra el día de la Victoria sobre la Alemania nazi cada 9 de mayo a lo largo y ancho de su territorio (de acuerdo con una encuesta del Centro Levada, un 75\% de los rusos tenían previsto celebrar la fiesta en $2010^{2}$ ). Hay, a pesar de ello, un centro privilegiado de la conmemoración, el desfile militar en la Plaza Roja de Moscú, un acontecimiento que es seguido masivamente por televisión (se emite en directo por los dos principales canales de Rusia, Pervi Kanal y Rossia 1). De hecho, según datos de la citada encuesta, un $79 \%$ de los rusos tenía previsto ver el desfile, y según los datos de TNS, la empresa encargada en Rusia del registro de las audiencias televisivas, la retransmisión del acontecimiento fue seguido por el $75,2 \%$ de los televidentes ${ }^{3}$. A tenor de lo que muestran estos datos podemos concluir que el desfile de la Victoria es el principal acontecimiento mediático de la actual Rusia, que tiene su punto culminante en la pronunciación del discurso presidencial, el lugar de articulación de la significación de la Victoria para los rusos. HutChings y RULYOva (2009: 139-55), afirman en su artículo sobre la celebración de la Victoria en 2005 que el discurso es un homenaje a los soldados y celebra la victoria del bien sobre el mal y de la libertad sobre el te-

1 Encontramos un ejemplo de la planificación temática en la conversión de San Petersburgo en ciudad heroica tras el bloqueo de 900 días al que estuvo sometida la ciudad durante la II Guerra Mundial comentada en MARTínez, Antonio, "El mito de San Petersburgo en el cine soviético a través de las adaptaciones de 'El capote' de Gógol", Comunicación y Sociedad, vol. XXIII, n 2, 2010, pp. 125-147

${ }^{2}$ Aktualnie voprosi prazdnovania Dnia Pobedi v Rossii [Cuestiones actuales de la celebración del Día de la Victoria en Rusia]. http://www.levada.ru/press/2010050401.html

${ }_{3}$ TNS - Audiencias de los medios (TV). http://www.tns-global.ru/rus/data/ratings/tv/index.wbp 
rror. A pesar de que, evidentemente, el discurso es, en gran medida, una expresión de agradecimiento a los veteranos por su sacrificio durante la Gran Guerra Patria, desde nuestro punto de vista es también una forma -quizás, la principal forma- de construcción de la identidad rusa surgida de las cenizas de la Unión Soviética a través del uso de las experiencias y los valores del pasado para levantar el edificio del presente y del futuro de Rusia y de los rusos.

\section{Objetivos}

El presente artículo se propone el análisis de la estructura profunda de los últimos once discursos presidenciales -desde el primer discurso de Vladímir Putin en el año 2000, hasta el discurso de Dmitri Medvédev en la conmemoración del 65 aniversario de la Victoria en 2010- con el fin de poner de manifiesto los recursos a través de los cuales el gobierno ruso construye su identidad nacional: los atributos de la nueva identidad rusa a partir del enlace del pasado y el presente, la definición de los nuevos enemigos de Rusia y la herencia que las nuevas generaciones han recibido de sus padres y abuelos soviéticos 65 años después del final de la guerra. La construcción de identidades ha sido uno de los objetos de estudio preferentes en el análisis de los medios, desde los estudios culturales (BARKER, 2000; HALL, 1996; MORLEY, 1995), el análisis del discurso (VAN DIJK, 1997), la interculturalidad (RODRIGO, 2000) o los acontecimientos públicos (HANDELMAN, 1990; MosSe, 1991) a aproximaciones teóricas (CASTELló, 2008) y sobre la relación entre los medios y el poder político (CASTELLS, 2001). Por lo que se refiere a las identidades rusa y soviética, las contribuciones a su análisis provienen del campo de la historia y la política (BitTman, 1989; Dunlop, 1985; Ferri, 2008, Medvédev, 2000; PoCH-DE-Feliu, 2003 o Hosking, 1983, entre otros), del análisis de la cultura y la literatura (Hutchings, 2006; MEAd, 2001; NivAT, 1993) y, finalmente, de los medios de comunicación y el cine (BEumers, 1999; Hutchings, 2009, Zassoursky, 2004; TURPIN, 1995). En cuanto a la construcción de la identidad rusa post-soviética a través de los medios, las contribuciones más relevantes son las obras de Stephen Hutchings sobre la conmemoración del 300 aniversario de la fundación de San Petersburgo (HuTCHINGS, 2008), y el análisis de la conmemoración televisiva de la Victoria en 2005 (Hutchings y Rulyova, 2009). A pesar de que, como ya hemos visto, en las obras de Hutchings se hace referencia a los discursos de la Victoria, no se realiza un análisis en profundidad de ellos, ni de lo que significan en la construcción identitaria del actual ciudadano ruso. El objetivo de este trabajo es utilizar la semiótica narrativa como herramienta para descifrar las claves de la construcción de la identidad rusa, fundamentalmente a través del otorgamiento de competencias a los ciudadanos $\mathrm{y}$, a partir de los resultados del análisis, proponer una metodología centrada en las competencias como un nuevo campo a explorar en el análisis de la relación entre discurso e identidad. Este artículo, pues, llena diversos vacíos en los estudios de comunicación en España. Por un lado, es pionero en el análisis del discurso político en Rusia y de la construcción identitaria tras la desaparición de la URSS, un tema no tratado en nuestro ámbito a pesar de su trascendencia en la configuración geopolítica europea de principios del siglo XXI. Por otro, pretende realizar un avance analítico a través de la modelización de la narración situando la competencia en el centro de los discursos y 
de la construcción de la identidad. La celebración en 2011 de los 20 años de la disolución de la Unión Soviética y del "año de Rusia" en España son, además, dos ocasiones oportunas para colocar el primer peldaño en el análisis de la política, los medios y la identidad en el que es el mayor país del continente europeo.

\section{Metodología}

Los discursos de la Victoria que analizamos a lo largo de este artículo son tratados como una unidad de análisis. Esto es, mediante una metodología que nos permita observar las invariantes que anidan en todos los discursos, tomados como unidad de significación aunque formalmente divididos en 11 ocurrencias o discursos particulares diferentes, proponemos una lectura unívoca de ellos. La metodología que usamos es la semiótica de corte estructuralista. Más exactamente, la parte de la semiótica estructural que se dedica al análisis y el estudio de los textos, entendiendo éstos como narraciones desglosables en categorías universales. Conocida como semiótica narrativa o análisis mio-narrativo, esta disciplina nos permite hallar a través de los textos una estructura de roles -los actantes o roles actanciales-, que se articula de manera similar en cada uno de los discursos. Así, construyendo una identificación entre discursos diferentes, las estructuras actanciales permiten afirmar algo así como: aquellos discursos que superficialmente parecen decir cosas diferentes, en realidad dicen lo mismo.

La semiótica narrativa, reafirmada por la prominencia del estructuralismo durante la segunda mitad del siglo XX, creció como subdisciplina de la semiótica y la narratología y profetizó que todo intercambio social, texto, u obra artística, en general, podía entenderse en términos de narración. El miembro más insigne de la Escuela de París, A. J. Greimas, retomó para tal fin el clásico de Vladimir Propp (1985), en el que el autor ruso había demostrado la reiteración de estructuras en el análisis de más de un centenar de cuentos fantásticos. Propp extrajo un total de 31 funciones y 7 caracteres que se repetían una y otra vez (HARTZ y STEGER, 2010: 769). Greimas complementó estos hallazgos y lo que en Propp eran figuras, es decir, personajes y actores que aparecían en el nivel superficial de los cuentos en forma de héroes, espadas o dragones, en Greimas se convirtieron en roles actanciales, posiciones vacías sin figurativizar, que no se experimentan directamente en el texto sino que residen en su nivel profundo y a las que hay que llegar a través de operaciones de abstracción.

Ha sido común en el estudio narratológico del siglo XX este esfuerzo por encontrar una estructura profunda que esté latente en el interior de cualquier tipo de discurso. Al estudio ya comentado de Vladimir Propp le siguieron autores como Etienne Souriau o Joseph Campbell. Souriau identificó en 1947 que ciertas iteraciones podían encontrarse en diferentes obras teatrales. Dos años después, Campbell lanzó la hipótesis de que todos los mitos, cualesquiera que sean las culturas en las que toman forma definitiva, responden a un patrón común que él denominó monomito (monomyth). En este sentido resulta reveladora la afirmación de Claude LeVI-Strauss (2006: 233): "Se puede definir al mito como ese modo del discurso en el que el valor de la fórmula traduttoretraditore tiende prácticamente a cero". Para Campbell toda narración puede reducirse al modelo de un héroe que inicia un viaje en donde atravesará diferentes umbrales, adquirirá competencias, realizará una determinada hazaña y regresará de nuevo a su hogar. 
Aquí reside la génesis de lo que más tarde Greimas llamará el "Esquema Narrativa Canónico". La búsqueda de este esquema se convirtió, en palabras de N.J. LowE, en el Santo Grial de la narratología de la segunda mitad del siglo pasado:

A more ambitious solution is to look for a deep structure underlying the production of individual story patterns. [...] the attempt to extend and generalize this procedure to the analysis of narrative as a whole is largely a phenomenon of the structuralist era, inspired in the fifties by the pioneer projects of Propp and Souriau, but driven by the new and initially attractive hypothesis that stories, like other kinds of mental and cultural structure, are organized and understood through syntactic mechanism similar to those of natural language. This quest for a workable grammar of fiction became the Grail of narrative theory throughout the 1960s and 1970s [...] (LowE (2000: 10).

Ya hemos señalado cómo el modelo greimasiano supone una evolución respecto a los modelos narrativos anteriores de Propp, Campbell o Souriau al proponer roles actanciales, posiciones vacías por llenar, en lugar de figuras. Consideramos que los discursos presidenciales de la Victoria admiten un análisis actancial y pueden abstraerse en una serie de roles que nos posibilitarán descifrar las relaciones entre ellos y sus diferentes funciones dentro de lo que denominamos el relato. Esta manera de proceder al análisis mediante los instrumentos de la semiótica narrativa nos permite seguir un método que es al mismo tiempo rastreable y verificable. Como recuerda el propio GREIMAS (2007: 91): "la superioridad metodológica de las lingüística estructural se basa en sus procedimientos de verificación". Es decir, que pone de manifiesto y al descubierto desde el principio todos los caminos utilizados para poder llegar a afirmar lo que se afirma, pudiendo así ser contradicho por ulteriores investigaciones.

La Victoria no es sólo un discurso verbal, es una celebración en la que se entrecruzan múltiples códigos semióticos y en donde espacios, personajes, acciones, objetos y música se funden con los discursos verbales formando un todo. En este artículo nos centraremos exclusivamente en los 11 discursos pronunciados por los dos presidentes rusos desde el año 2000 al 2010. La justificación de esta delimitación de nuestro corpus de estudio es doble. Por un lado, estamos con GrEIMAS y COURTÉs (2006: 230) cuando definen la isotopía como "la iteratividad -a lo largo de una cadena sintagmática- de clasemas que aseguran al discursos-enunciado su homogeneidad". Es decir, que aunque por un lado los diferentes códigos puestos en circulación en el texto llamado "Día de la Victoria" comunican a través de canales diferentes, cabe pensar, como así se demostrará, que todos ellos funcionan/significan en un mismo sentido, homogéneamente. No existe contradicción entre la significación de uno de esos códigos y el resto; por tanto, se puede concluir que la significación hallada en el discurso verbal del presidente se rige por el principio de isotopía y refrenda el resto de códigos. Por otro lado, la segunda razón para la delimitación del corpus al discurso verbal del presidente podríamos resumirla en unas palabras de Roland BARTHES recogidas por FABBRI (2000: 24): "todos estos sistemas de significación son comprensibles y traducibles en el sistema supremo y extremo de signos que es la lengua [...] A diferencia de otros sistemas (visual, gestual, musical, espacial, etc.) la lengua es capaz de nombrarse a sí misma y a los otros signos de la cultura". 
Al margen del Modelo Actancial contamos con un segundo instrumento de apoyo para extraer la significación de los discursos. Se trata del cuadro semiótico, cuya utilidad queda demostrada en investigaciones recientes (KOLZINETS, 2008: 865-881; FISHER, 2005: 239-255). La proyección del cuadro semiótico sobre algunas de las categorías principales de los discursos estudiados aporta una información relevante sobre la manera de construirse de estos textos. Según Floch (2000:117), la definición del cuadro semiótico sería "a representation -both static and dynamic- of the first articulations of meaning (sens). It is a topography of the relationships and the processes that constitute the minimal conditions for the production of meaning (signification) [...]". Tomando como punto de partida esta definición, el cuadro semiótico permite comprender cómo los discursos de la Victoria llevan a cabo la construcción de la identidad nacional rusa.

\subsection{Modelo Actancial}

Como ya se ha mencionado, la identificación de la estructura narrativa de los discursos presidenciales es nuestra principal herramienta de análisis. El Modelo Actancial distingue 6 roles actanciales básicos: el Destinador del Contrato (DC), el Sujeto de Acción (S), el Sujeto de Estado (SE), el Objeto de Valor (O), el Adyuvante (Ad) y el Oponente (Op). Con el fin de resumir el tipo de estructuras que pueden crear estos roles, podemos concretar sus funciones de la siguiente manera: el Destinador del Contrato (DC) encarga una misión al Sujeto de Acción (S), al que ha manipulado ${ }^{4}$, para que éste realice la acción. Esta misión consiste en que el Sujeto de Estado (SE) establezca algún tipo de relación con el Objeto de Valor (O). Para conseguir tal fin el Sujeto de Acción (S) contará con la ayuda de Adyuvantes (Ad) y los obstáculos de los Oponentes (Op).

En relación a estos 6 roles actanciales que acabamos de presentar nos gustaría introducir el concepto de competencia (C), que resulta de gran utilidad para encarar los análisis semio-narrativos. En narratología la competencia es aquello que el S necesita tener o adquirir para llevar a cabo con éxito la misión que se le ha encomendado. En el caso de los discursos presidenciales es un concepto clave para entender su significado. Como concretamos más adelante, una de las hipótesis de partida de la investigación se refiere al hecho de que entendemos que los discursos de la Victoria son principalmente un mecanismo de transmisión de competencias al pueblo de Rusia. Mediante la narración que llevan a efecto los discursos, el auditorio recibe una serie de habilidades y capacidades provenientes del pasado. Sin entrar todavía en estos pormenores, nos gustaría aclarar que el concepto de $\mathrm{C}$ tiene su contrapunto en el de atributo. Dentro del Modelo Actancial existen unos roles activos, es decir, que realizan acciones -el DC, el S, el Ad y el Op.- y otros pasivos y que, por tanto, sólo reciben o sufren la acción del resto de roles -el O y el SE-. De este modo, parece oportuno dis-

4 Como se afirma en Zunzunegui (2003: 35): "la comunicación debe entenderse menos como un hacer-saber que como un hacer-creer o, si se prefiere, un hacer-hacer, lo que implica el poner en primer término su carácter de manipulación, entendida como una "acción del hombre sobre otros hombres para hacerles ejecutar un programa dado". 
tinguir entre las cualidades que un rol activo posee, las competencias, y las que posee un rol pasivo, los atributos.

\section{Análisis de los discursos presidenciales}

Los discursos de la Victoria admiten muchas aproximaciones desde una misma perspectiva metodológica. Independientemente del acercamiento que se escoja, podemos aseverar que la Victoria es un discurso persuasivo que busca una finalidad, cuando menos de tipo comunicativo, en donde el emisor del discurso tiene por objetivo que su público adquiera cierta información y determinado enfoque sobre ella. De acuerdo a la teoría de los actos del habla (AUSTIN: 2004) esta primera instancia del discurso correspondería a los actos locutivos -en donde se expresa la idea del mensaje- e incluiría actos ilocutivos -una finalidad u objetivo para conmover o emocionar al auditorio-. En cualquier caso, sin obviar ninguna de estas dos vertientes del discurso, nos parece más oportuno abordar la Victoria como una narración o acto del habla perlocutivo en donde al hablar se realiza una acción: la comunicación es la acción-. De la misma manera que en los ejemplos clásicos de este tipo de actos performativos un "sí, quiero" significa la acción de casarse, un "declaro la guerra" significa iniciar una guerra, en los discursos de la Victoria los actos comunicativos devienen acciones en sí: un recuerdo se convierte en el acto de homenajear y así la memoria no es un hacer-pasivo sin vida actual sino una llamada a la acción y un acto de arenga y de transmisión de competencias desde el pasado al presente.

Vistos los discursos desde este prisma, debemos identificar dos estructuras narrativas distintas en el texto. Si, como afirmamos, el discurso consiste en la transferencia de unas competencias desde unos actores del pasado a unos actores del presente y futuro, se hace necesario identificar cuáles son estas dos estructuras a través de las cuales se articula tal transferencia. Tendremos de este modo un primer Modelo Actancial del pasado $\left[\mathrm{T}_{1}\right]$, mediante el cual el Presidente ruso construye la narración de los hechos que condujeron al triunfo soviético y aliado en la II Guerra Mundial.

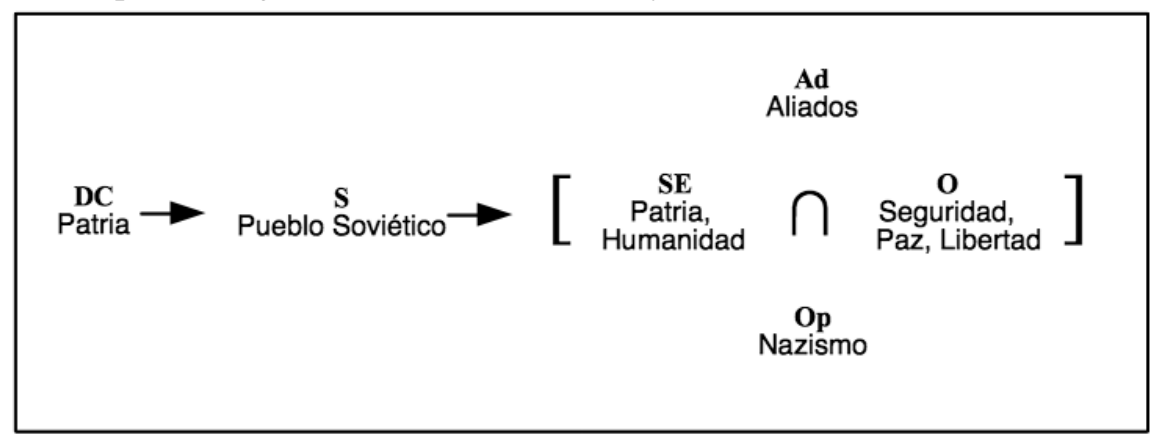

FIGURA 1. Modelo Actancial [ $\mathbf{T}_{\mathbf{1}}$ ]

En $\mathrm{T}_{1}$ podemos leer la narración del siguiente modo: la madre patria, la URSS, encarga a sus ciudadanos, el pueblo soviético, que realicen una misión; esta misión consiste, por un lado, en que la patria se mantenga segura, libre y en paz de los enemigos que la acechan y por otro lado en que la Humanidad en su conjunto consiga estos mis- 
mos objetivos. Para conseguir el éxito en esta misión cuenta con la ayuda de las fuerzas aliadas, que luchan junto a ellos en la Segunda Guerra Mundial. E esta misión es obstaculizada por los nazis, que tratan de arrebatarles la libertad, seguridad y paz que persiguen.

El discurso presidencial recupera de esta estructura actancial de $\mathrm{T}_{1}$ aquellos conceptos o valores que quiere aplicar al discurso sobre el presente. Mediante un proceso de identificación de situaciones los acontecimientos del presente se presentan como el eterno retorno de lo mismo. Los discursos de la Victoria operan bajo la presunción de partida de que la situación presente $\left(\mathrm{T}_{2}\right)$ es, en gran medida, equivalente o paralela a la situación pasada $\left(\mathrm{T}_{1}\right)$. Así, los presidentes Putin y Medvédev construyen unos relatos en donde misiones, ayudantes, oponentes, amenazas y objetos de $\mathrm{T}_{1}$ se vuelven a presentar ahora, $\mathrm{T}_{2}$, bajo nuevos nombres y caras -quí reside precisamente la idoneidad del método semio-narrativo, ya que permite profundizar más allá de la figurativización de superficie del relato y acceder a las instancias profundas que comparten los relatos de $\mathrm{T}_{1} \mathrm{y}_{7}$-- - pero con idéntica función.

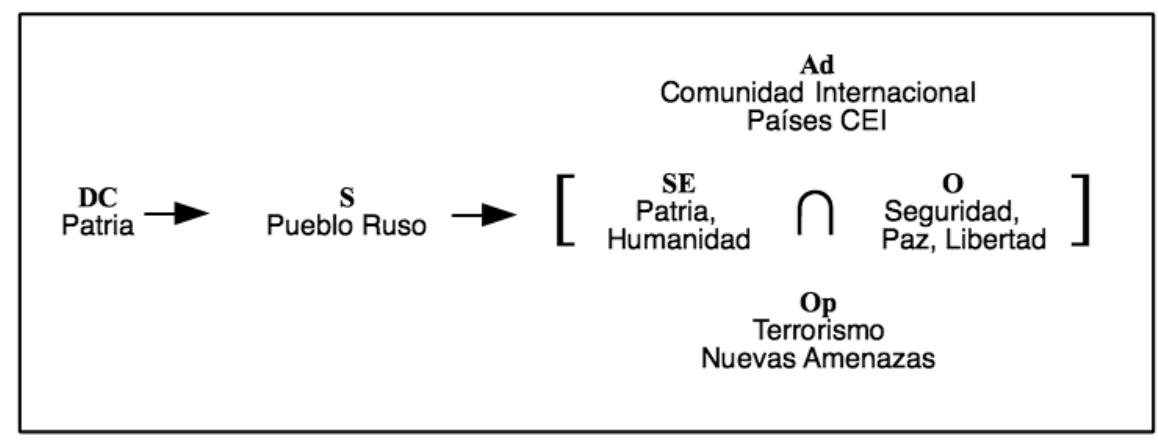

FIGURA 2. Modelo Actancial [ $\left.\mathbf{T}_{2}\right]$

Consecuentemente, en $\mathrm{T}_{2}$ podemos leer la narración del siguiente modo: la madre patria, Rusia, encarga a sus ciudadanos, el pueblo ruso, que realicen una misión; como en $\mathrm{T}_{1}$, esta misión consistirá: por un lado, en que la patria se mantenga segura, libre y en paz de los enemigos que la acechan y; por otro lado, en que la Humanidad en su conjunto consiga estos mismos objetivos. A diferencia de $\mathrm{T}_{1}$, para conseguir el éxito en esta misión debe contar con la ayuda de la Comunidad Internacional y de los países de la Comunidad de Estados Independientes (CEI), que lucharán junto a ellos contra el nuevo enemigo. La misión se verá obstaculizada por las amenazas contemporáneas, que tratarán de arrebatarles la libertad, seguridad y paz que persiguen. Estas amenazas toman diferentes formas en $\mathrm{T}_{2}$ : terrorismo, terrorismo internacional, y todo aquel que pretenda poner en peligro la unidad y la integridad de Rusia.

\subsection{Las competencias de los actantes}

Christel Lane afirmaba respecto al análisis de los rituales soviéticos que "to create symbolism from words is a very difficult task and requires great restraint and imagination. To become symbolic, a verbal construction must propel its listeners into action" 
(LANE, 1981: 201). Los discursos presidenciales son construcciones simbólicas verbales que realizan una llamada a la acción de la audiencia, tanto en el plano indicativo como en el subjuntivo, que podríamos resumir como el combate contra los nuevos enemigos y la conservación y defensa de lo que hemos definido como principales objetos de valor: la seguridad, la paz y la libertad. Con el fin de comprobar de qué manera la narración realiza esta llamada a la acción, nos fijaremos en las competencias de los actantes, los requisitos indispensables para que lleven a término con éxito sus acciones.

Del análisis de los once discursos podemos extraer cuáles son las competencias del sujeto de acción en $\mathrm{T}_{1}$ que le permitieron finalizar con éxito la misión de conseguir la victoria sobre el régimen nazi. El pueblo soviético tenía audacia, coraje, dignidad, fe, firmeza, fuerza de espíritu, fuerza de voluntad, generosidad, heroicidad, honor, patriotismo, poder, resistencia, responsabilidad, solidaridad, unidad y voluntad de sacrificio. Todas ellas eran condiciones indispensables para combatir a un enemigo poderoso, el nazismo, que tenía las competencias de arrogancia, brutalidad, crueldad, destrucción, fuerza, intolerancia, invasión, monstruosidad o tortura. Finalmente, respecto al tercero de los actantes activos, los adyuvantes, la principal competencia que se deriva de los discursos es la unión.

Si estas son las competencias de los actantes activos en $\mathrm{T}_{1}$, las de los actantes en $\mathrm{T}_{2}$ resultan de un proceso de transferencia de éstas a través de dos vías: (1) la sangre y (2) la memoria, entendiendo aquí la memoria como la identificación histórica a través del recuerdo entre los actantes en $\mathrm{T}_{1}$ y los actantes en $\mathrm{T}_{2}$. En el caso de $\mathrm{S}$, las competencias son transferidas por ambas vías. En cuanto a la transferencia por vía sanguínea, las nuevas generaciones de rusos llevan la Victoria "en la sangre" (Putin, 2000), la herencia de los combatientes en la Gran Guerra Patria está en su sangre y las hazañas en sus corazones (PUTIN, 2001), la dignidad y la unidad de la generación de los que combatieron al nazismo es la herencia más valiosa que han recibido sus hijos y nietos (Putin, 2002) y a todos los rusos les ha sido dada una valiosa experiencia que les "enseña a luchar hasta la victoria y ser fuerte ante las dificultades y asumir la responsabilidad con uno mismo y con su pueblo" (MEDVÉDEv, 2009) y una gran reserva de fe en el triunfo de la verdad, en la fuerza, la independencia y la libertad que abren a muchas generaciones una "época de proezas y victorias pacíficas" (MEDVÉDEV, 2008). La transferencia por vía de la memoria es, a diferencia de la sanguínea, una acción que, a la vez realiza el discurso -y el conjunto de actos conmemorativosy la audiencia, los ciudadanos rusos, que deben conservar la memoria de la Gran Victoria y las hazañas de sus padres y abuelos, para defender los intereses de la patria (Putin, 2007). En consecuencia, el pueblo ruso es un pueblo competente para luchar y vencer como lo hizo el pueblo soviético durante la Gran Guerra Patria ya que, a través de la sangre y la memoria, conserva las competencias de éste último.

En el caso del Op, la transferencia de sus competencias ya no se realiza a través de la sangre, sino a través de la memoria, atribuyendo a los nuevos enemigos las mismas capacidades que al régimen nazi. Este nuevo enemigo, que tiene diferentes formas en función del contexto político, es un enemigo "no menos terrible" que el nazismo, "lleva la muerte y la destrucción” (PuTIN, 2004) y “como en la época del tercer Reich, 
sienten el mismo menosprecio a la vida humana" (Putin, 2007). Las amenazas, pues, son las mismas que en la época del nazismo, "sólo se han transformado, han cambiado de aspecto" (Putin, 2007). En consecuencia, el enemigo actual no sólo es tan poderoso y destructor como el nazismo, sino que añade una competencia nueva, su mutabilidad y la dificultad de su identificación unívoca: el terrorismo, el terrorismo internacional, los que pretenden redefinir las fronteras o no cumplir con la legalidad internacional y, en general, cualquiera que pretenda poner en peligro la vida y la memoria de los rusos y la unidad y la integridad territorial de Rusia.

Finalmente, la unión, la principal de las competencias de los adyuvantes, también es transferida a través de la memoria. En los discursos se afirma que, como demostró la coalición antihitleriana, "sólo con la unión de los esfuerzos de los estados y la voluntad de las naciones se pueden combatir estas amenazas" (PUTIN, 2002), una unidad y una hermandad fortalecida en la lucha contra el nazismo (PuTIN, 2004), una unidad basada en el pasado y dirigida a un futuro común (PuTIN, 2005), y que es el testigo de una disposición común a la defensa de la paz (MEDVÉDEV, 2010).

\subsection{El programa narrativo de enlace. La memoria y el paso del tiempo}

El hecho de que la memoria transfiera competencias de los diferentes actantes activos de $\mathrm{T}_{1}$ a $\mathrm{T}_{2}$ nos permite asumir un segundo esquema narrativo en $\mathrm{T}_{2}$ en el que el objeto (memoria) es una condición previa para el enlace entre Modelo Actancial $\left(\mathrm{T}_{1}\right)$ y Modelo Actancial $\left(\mathrm{T}_{2}\right)$. Si no recordamos qué hicieron $\mathrm{S}\left(\mathrm{T}_{1}\right), \mathrm{Op}\left(\mathrm{T}_{1}\right)$ y $\operatorname{Ad}\left(\mathrm{T}_{1}\right)$ no podremos heredar sus competencias:

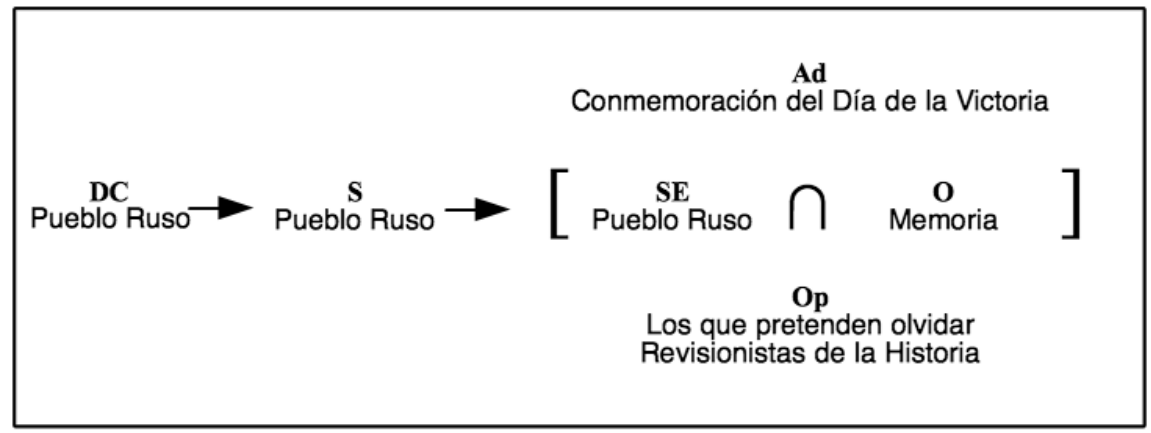

FIGURA 3. Modelo Actancial [Enlace de memoria de $\mathbf{T}_{\mathbf{1}}$ a $\mathbf{T}_{\mathbf{2}}$ ]

En los discursos de la Victoria analizados son frecuentes las menciones a los años pasados, a la necesidad, a la obligación al recuerdo (Putin, 2003) y al no derecho a olvidar (Putin, 2006). Según la versión de los discursos, el tiempo pasado es mucho, pero se recuerda todo (Putin, 2000). Rusia debe conservar siempre la memoria de la Victoria (Putin, 2007), la dureza de los años de la Gran Guerra Patria no deben olvidarse jamás, pasen los años que pasen (MEDVÉDEV, 2008), y, a pesar del poder enorme del tiempo, "es más débil que la memoria humana, que nuestra memoria" (MEDVÉDEv, 2010). 
Uno de los problemas a los que se enfrenta el Estado ruso es el alejamiento de las generaciones más jóvenes respecto a los hechos de la Gran Guerra Patria y la Victoria. Como afirma Yákov GoRDIN:

En realidad, a la juventud actual no le interesan los acontecimientos de la Gran Guerra Patria. Para ellos, la guerra forma parte de un pasado histórico y lejano. No es nada sorprendente. Cuando nosotros éramos jóvenes también nos resultaban lejanos los acontecimientos de la guerra de Turquía de 1877-1879 y la guerra con Japón de 1904-1905 (GoRDIN, 2005: 5).

Según una encuesta del Centro Levada ${ }^{5}$ realizada en 2010, los jóvenes rusos entre 18 y 24 años son los que menos miraron el desfile militar conmemorativo de la Victoria (el $31 \%$ afirmaba no haberlo visto, frente al $18 \%$ de los mayores de 55 años) y a los que menos gustó el acto (gustó al $62 \%$ de los jóvenes, frente al $70 \%$ de media). LANE (1981) ya exponía esta misma preocupación de los dirigentes soviéticos respecto a la pérdida de contacto con las tradiciones de la Revolución y el Trabajo a partir de la década de 1970. Lo mismo sucede en la Rusia actual, en la que el Estado ruso realiza enormes esfuerzos para evitar que las nuevas generaciones pierdan el contacto con la Victoria y sus tradiciones (claro ejemplo de ello son el impulso, desde 2005, de la acción de la cinta de San Jorge, prácticamente omnipresente en las actuales celebraciones de la Victoria y definida por sus creadores como una iniciativa para "unir el día de la celebración de la Victoria a gente de diferente edad y condición social" y el lema de la cual es "Yo lo recuerdo, yo me enorgullezco", o la página web del presidente de la Federación de Rusia en el que se explica a los niños rusos por qué se celebra el Día de la Victoria ${ }^{7}$, entre muchas otras acciones que tienen como finalidad común la perpetuación de la memoria a través de la adhesión de las nuevas generaciones). Como ya hemos visto, en los discursos se manifiesta claramente esta preocupación. En la mayoría de ellos se mencionan los años pasados; pero si en un principio el tiempo debería ser un enemigo de la memoria y, por lo tanto, del enlace entre la generación que combatió en la guerra y la generación actual, el discurso potencia este enlace afirmando, por ejemplo, que "cuanto más lejos quedan los hechos de esta terrible guerra, más valiosa es nuestra hermandad y solidaridad seculares" (MEDVÉDEV, 2008), o bien atribuyendo al tiempo unas competencias inferiores a las de la memoria, afirmando que "el tiempo tiene un poder enorme" pero "es más débil que la memoria humana” (MEDVÉDEv, 2010). Además, del análisis de los discursos observamos que, cuanto más nos alejamos de los hechos conmemorados, más se menciona el paso del tiempo y el poder de la memoria sobre él.

5 Rossiane o prazdnovanii Dnia Pobedi [Los rusos sobre la celebración del Día de la Victoria] http://www.levada.ru/press/2010061802.html

6 Vsiá pravda o "Gueoruievskoi lentochke" [Toda la verdad acerca de la "Cinta de San Jorge"] http://www.rian.ru/spravka/20070423/64190392.html

7 Pochemu my prazdnuiem Den Pobedi [¿Por qué celebramos el Día de la Victoria?] http://kids.kremlin.ru/index.php? fw $=11 \& \mathrm{p}=5-6-8 \& \mathrm{v}=\mathrm{fi} 12$ 
En relación con el tiempo, los discursos presidenciales enfatizan el carácter eterno de la Victoria y de su transmisión generacional: el pueblo ruso siempre recordará la hazaña de los veteranos (Putin, 2007), siempre recordará la ayuda de los aliados y la fiesta de la Victoria siempre será sagrada y recordada (PuTin, 2005), el 9 de mayo siempre será el día de la liberación del mundo, "el día en que empezó un nuevo periodo de la historia universal" (MEDVÉDEV, 2008). En este mismo sentido, los discursos también enfatizan el carácter eterno del tributo a los veteranos y de la memoria del pueblo ruso. Incluso a la conmemoración de la Victoria se le atribuye ese carácter, cuando se afirma que es la fiesta "que siempre ha sido y será la más popular, la más sagrada y se ha convertido para siempre en el símbolo de nuestra unidad nacional” (MEDVÉDEV, 2008). En este último caso, vemos una proyección tanto al tiempo pasado -desde siempre-, como al futuro -para siempre-, hecho que se traduce en una continuación ad eternum del proceso de transferencia analizado en este trabajo, a pesar de basarse en una afirmación engañosa, sobre todo si consideramos que las otras dos tradiciones sagradas soviéticas eran, hasta el final de la URSS, las más celebradas en Rusia.

\section{Discusión}

Uno de los mayores retos al que se enfrentan los emisores del discurso de la Victoria consiste en superar la desafección que las nuevas generaciones de rusos padecen hacia los hechos que la Victoria describe. Esta debilidad es contrarrestada por el discurso a través de una estrategia constante: convertir en activo todo aquello que es pasivo. Los hechos de 1945 no pueden ser percibidos como algo perteneciente al pasado y sin vigencia actual. Visto así, el texto opera una serie de transformaciones por las cuales conceptos pasivos como "pasado", "olvidar" o "sufrimiento" son convertidos en conceptos activos como "futuro", "recordar" o "lección". De esta manera, y como forma general de entender las operaciones de construcción de identidad nacional que el texto propone, las nuevas generaciones son espoleadas a la acción mediante el paso, en el discurso, de sus atributos pasivos -su relación sanguínea con sus antepasados- a sus competencias activas -la memoria y la lección aprendida con usos en el futuro-.

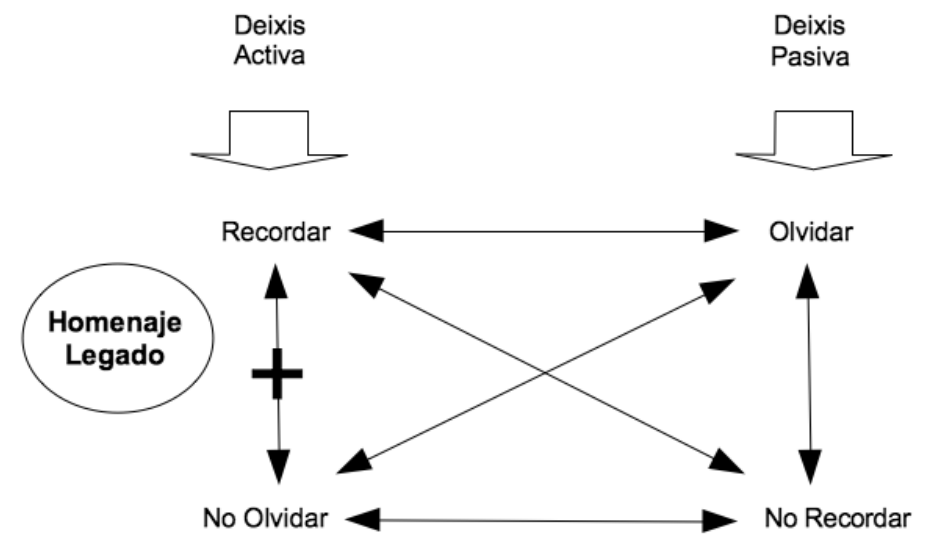

FIGURA 4. Recordar-Olvidar 
Como apreciamos en el cuadro, se opera un vertimiento desde la deixis pasiva aquella que se intenta evitar y cuyos peligros son el paso del tiempo y la desafección de las nuevas generaciones, en una palabra: el olvido -hacia la deixis activa del cuadro- lugar del homenaje y el legado, que a través del deber de recordar y la obligación de no olvidar perpetúa la identidad en el pueblo ruso al no permitir una pérdida de las competencias adquiridas.

La transmutación de lo pasivo en activo funciona en múltiples niveles de la narración. Una característica destacable de los discursos es la confusión constante entre el individuo como ciudadano y como soldado. La Victoria es a veces atribuida al ejército y otras a los ciudadanos; lo mismo ocurre con la herencia de la tradición de la Victoria o las nuevas misiones que la patria les encarga. La Victoria en $T_{1}$ es la suma de los esfuerzos tanto de los hombres que lucharon en el frente como de las mujeres que sustituyeron a sus maridos en las fábricas o asistieron a los enfermos en los hospitales. Si $\mathrm{S}$ en $\mathrm{T}_{1}$ es un sujeto colectivo en donde ejército y ciudadanía son inseparables, $\mathrm{S}$ en $\mathrm{T}_{2}$ hereda estas mismas competencias y niega que pueda construirse una identidad nacional basada en un ejército activo y una población pasiva.

En este sentido, el análisis semiótico pone al descubierto un aspecto básico de los discursos presidenciales de la Victoria: la narración muestra como innecesaria la adquisición de competencias, ya que el pueblo ruso las posee y le son transferidas por vía sanguínea, pasivamente, y vía memoria, activamente. Los discursos sirven para comunicar a los ciudadanos rusos que saben y pueden vencer, que son herederos de quienes ya lo hicieron, pero que su Victoria nunca es definitiva. Rusia necesita construir un relato en el cual los objetos de valor conseguidos sean valores en tensión, en peligro constante de volver a perderse/olvidarse. Los rusos deben luchar constantemente por nuevas victorias porque los enemigos están siempre dispuestos a poner en peligro la unidad y la integridad de la patria, así como a revisar su Historia y socavar su memoria. Una memoria que, cabe decir, es una memoria selectiva, que sólo recoge los hechos y las características afirmativas de los rusos, y que olvida no sólo errores, complicidades y atrocidades propias, sino que borra del todo la huella de algunas de las causas por las que los ciudadanos soviéticos se lanzaron a la lucha contra el nazismo ${ }^{8}$.

\section{Conclusión}

Gayle Hollander inventarió las principales cualidades del ciudadano soviético, entre las que estaban el amor al trabajo, la politización y la subordinación de sus emociones, intereses y valores a los objetivos comunes, la vigilancia hacia los "enemigos internos" -familia y amigos incluidos-, la solidaridad con los trabajadores de todo el mundo, el patriotismo, el virtuosismo o el ateísmo (Cfr. Hollander, 1972:8). La identidad soviética se basaba en la relación del individuo con el trabajo, la política, la clase, la patria, la moral y la religión. De los discursos de la Victoria podemos extraer las características atribuidas al ciudadano ruso, relacionadas con las competencias

8 En la obra Parad Pobedi podemos comprobar como, al menos hasta 1985, la victoria sobre el régimen nazi era atribuida al socialismo. "[...] El 9 de mayo demuestra la fuerza indestructible del socialismo y, a su vez, recuerda la vigilancia con respecto a las peligrosas intenciones de los agresores imperialistas y de sus cómplices” en DROZDOV y RIABKO (1985: 5). 
otorgadas y las obligaciones de la memoria, que enlazan con algunas de las cualidades descritas por Hollander -sobre todo las que tienen relación con la cultura patriótico-militar-. De este modo, las categorías en las que podemos diferenciar la configuración de su identidad son:

a) Respeto a la memoria: el ciudadano ruso respeta y es fiel a la memoria de los caídos y a las hazañas de los que sacrificaron sus vidas por la soberanía del país. Siente dolor por los que no sobrevivieron a la guerra y sabe, recuerda y reconoce -y cada vez más (MEDVÉDEv, 2008)- el precio de la Victoria. No tiene el derecho a olvidar ni a traicionar los santuarios y las banderas de la Victoria. Agradece y rinde homenaje a la generación victoriosa y tiene una deuda impagable con ella.

b) Defensa de la patria: el ciudadano ruso honra su patria y, como los veteranos, la defiende con abnegación -"la defensa de la Patria es nuestra obligación sagrada, es la base moral para todas las generaciones"- (MEDVÉDEV, 2009).

c) Heredero de la Victoria: el ciudadano ruso ha recibido la gran experiencia de la Victoria y de los veteranos, y porta la costumbre de vencer en sus venas, es heredero de sangre de la Victoria y reconoce en ella un honor, un orgullo y una responsabilidad. Ha aprendido a vencer en nombre de la patria.

d) Transmisión de la memoria: celebra la fiesta de la Victoria como propia, conserva y traspasa a sus descendientes la experiencia y la memoria, los valores y la moral de la Victoria. Ayuda a conservar y aumentar las tradiciones de la Victoria.

e) Obligación a nuevas Victorias: está obligado a nuevas victorias, a trabajar honradamente por la libertad, la prosperidad, el bienestar, la fortaleza y la paz de su país. Debe vencer y ser el primero en todo, realizar los actos morales más elevados. Debe trabajar para que sus éxitos sean dignos de los éxitos de los veteranos. Aunque sus ciudadanos vivan tiempos de paz, sus competencias heredadas y la importancia de la conservación de los valores que componen la identidad nacional rusa hacen que sea necesario estar en todo momento preparados para la guerra.

\section{Referencias bibliográficas}

AUSTIN, John (2004): Cómo hacer cosas con palabras: palabras y acciones. Barcelona, Paidós.

BARKER, Chris (2000): Televisión, globalización e identidades culturales, Barcelona, Paidós.

BEUMERS, Birgit (1999): Russia On Reels: The Russian Idea in Post-Soviet Cinema. New York, I.B. Tauris.

BITTMAN, Ladislav (1989): "Gorbachev's Glasnost: Challenges and Realities". Propaganda Disinformation Persuasion, vol. 2, $\mathrm{n}^{\circ}$ 4, Primevera. Boston, Boston University College of Communication Program for the Study of Disinformation Papers.

CAMPBELL, Joseph (1993): The Hero With a Thousand Faces. London, Fontana Press. 
CASTELLÓ, Enric (2008): Identidades mediáticas. Barcelona, UOC.

CASTELLS, Manuel (2001): La Era de la información. El poder de la identidad. Madrid, Alianza.

CASTELLS, Manuel (2001): La nueva revolución rusa. Madrid, Sistema.

DROZDOV y RIABKO (1985): Parad Pobedi [El desfile de la Victoria]. Moscú.

DUNLOP, John B. (1985): The New Russian Nationalism. New York. Praeger.

FABBRI, Paolo (2000): El giro semiótico. Barcelona, Gedisa.

FERRI, Llibert (2008): L'esclat de l'est. Universitat de Vic, EUMO.

FISHER, Colin (2005): "HRD Attitudes: Or the Roles and Ethical Stances of Human Resource Developers". Human Resource Development International, vol. 8 (2), pp. 239-255. UK, Nottingham Business School, Nottingham Trent University.

FLOCH, Jean-Marie (2000): Visual Identities. London, Continuum.

GORDIN, Yákov, Nasha Voiná (2005): [Nuestra guerra], San Petersburgo, Zvezdá.

GREIMAS, Algirdes Julien (2007): Semántica estructural. Investigación metodológica. Madrid, Gredos.

GREIMAS, Algirdes Julien; COURTÉS, Joseph (2006): Semiótica, Diccionario razonado de la teoría del lenguaje. Vol 1. Madrid, Gredos.

HALL, Stuart (1996): "Introduction: Who Needs 'Identity?", en HALL, Stuart y DU GUY, Paul (eds.): Questions of Cultural Identity. London, Sage.

HANDELMAN, Don (1990): Models and Mirrors. Cambridge University Press.

HARTZ, Ronald y STEGER, Thomas (2010): "Heroes, villains and 'honourable merchants': narrative change in the German media discourse on corporate governance". Organization 17, p. 767.

HOLLANDER, Gayle Durham (1972): Soviet Political Indoctrination. Developments in Mass Media and Propaganda Since Stalin. New York, Praeger Publishers.

HOSKING, Geoffrey A (1993): Empire and nation in Russian history. Waco, Baylor University, Markham Press Fund.

HUTCHINGS, Stephen C. (2006): Russian Modernism: The Transfiguration of the Everyday, Cambridge University Press.

HUTCHINGS, Stephen (2008):. Saint Petersburg 300: Television and the Invention of a Russian (Media) Tradition. A: Television New Media, 9 (3).

HUTCHINGS, Stephen (2009): Television in Putin's Russia. London, Routledge.

HUTCHINGS, Stephen, RULYOVA, Natalia (2009): “Commemorating the Past/Performing the Present: television coverage of the Second World War victory celebrations and the (de)construction of Russian nationhood", en BEUMERS, Birgit; HUTCHINGS, Stephen; RULYOVA, Natalia (eds): The Post-Soviet Russian Media: Conflicting Signals. London, Routledge, pp. 137-155. 
KOZINETS, Robert (2008). "Technology/Ideology: How Ideological Fields Influence Consumers' Technology Narratives". Journal of Consumer Research: An Interdisciplinary Quarterly, University of Chicago Press, vol. 34(6), pp. 865-881, October.

LANE, Christel (1981): The rites of rulers. Ritual in industrial society-the Soviet case. Cambridge University Press.

LEVI-STRAUSS, Claude (2006): Antropología estructural. Madrid, Siglo XXI.

LOWE, North J. (2000): The Classical Plot and the Invention of Western Narrative. Cambridge, Cambridge University Press.

MARTÍNEZ, Antonio (2010): "El mito de San Petersburgo en el cine soviético a través de las adaptaciones de 'El capote' de Gógol”. Comunicación y Sociedad, vol. XXIII, nº 2, pp. 125-147

MEAD, Margaret; GORER, Geofrey; y RICKMAN, John (2001): Russian culture. New York, Berghahn Books.

MEDVEDEV, Roy (2000): Post-Soviat Russia. A Journey Through the Yeltsin Era. New York, Columbia University Press.

MEDVEDEV, Dmitry (2008): Speech at Military Parade in Honour of 63th Anniversary of Victory in Great Patriotic War, 9 de mayo de 2008. Versión online:

http://archive.kremlin.ru/eng/speeches/2008/05/09/1642_type127286_200449.shtml

MEDVEDEV, Dmitry (2009): Speech at Military Parade in Honour of 64th Anniversary of Victory in Great Patriotic War, 9 de mayo de 2009. Versión online:

http://archive.kremlin.ru/eng/speeches/2009/05/09/1501_type127286_216101.shtml

MEDVEDEV, Dmitry (2010): Speech at the Military Parade to Commemorate the $65^{\text {th }}$ Anniversary of the Victory in the Great Patriotic War, 1941-1945, 9 de mayo de 2010. Versión online:

http://archive.kremlin.ru/eng/speeches/2010/05/09/1032_type127286_225909.shtml

MORLEY, David, ROBINS, Kevin (1995): Spaces of Identity. Global Media, Electronic Landscapes and Cultural Boundaries. London, Routledge.

MOSSE, George (1991): The nationalization of the masses. Political Symbolism and Mass Movements in Germany from the Napoleonic Wars through the Third Reich. EUA, Cornell University Press.

NIVAT, Georges, (1993): Russie-Europe. La fin du schisme. Lausana, Études littéraires et politiques. L'Age d'Homme.

POCH-DE-FELIU, Rafael (2003): La gran transición. Rusia, 1985-2002. Barcelona, Crítica.

PUTIN, Vladimir (2000): Address at a Parade Dedicated to the 55th Anniversary of Victory in the Great Patriotic War, 9 de mayo de 2000, versión online: http://archive.kremlin.ru/eng/speeches/2000/05/09/0001_type127286_85157.shtml 
PUTIN, Vladimir (2001): Speech at the Parade Celebrating the 56th Anniversary of Victory in the Great Patriotic War, 9 de mayo de 2001, version online: http://archive.kremlin.ru/eng/speeches/2001/05/09/0001_type82912type127286_8 5159.shtml

PUTIN, Vladimir (2002): Speech at the Military Parade Celebrating the 62nd Anniversary of Victory in the Great Patriotic War, 9 de mayo de 2002, versión online: no disponible.

PUTIN, Vladimir (2003): Speech at the Parade in honour of the 58th Anniversary of Victory, 9 de Mayo de 2003, versión online: http://archive.kremlin.ru/eng /speeches/2003/05/09/0001_type127286_44481.shtml

PUTIN, Vladimir (2004): Speech at the Military Parade Commemorating the 59th Anniversary of Victory in the Great Patriotic War, 9 de mayo de 2004, versión online: http://archive.kremlin.ru/eng/speeches/2004/05/09/1000_type127286_64263 .shtml

PUTIN, Vladimir (2005): Speech at the Military Parade in Honour of the 60th Anniversary of Victory in the Great Patriotic War, 9 de mayo de 2005, versión online: http://archive.kremlin.ru/eng/speeches/2005/05/09/1040_type82912type127286_8 7820.shtml

PUTIN, Vladimir (2006): Speech at the Military Parade Celebrating the 61st Anniversary of Victory in the Great Patriotic War, 9 de mayo de 2006, versión online: http://archive.kremlin.ru/eng/speeches/2006/05/09/1341_type127286_105494.shtml

PUTIN, Vladimir (2007): Speech at the Military Parade Celebrating the 62nd Anniversary of Victory in the Great Patriotic War, 9 de mayo de 2007, versión online: http://archive.kremlin.ru/eng/speeches/2007/05/09/1432_type82912type127286_1 27675.shtml

PROPP, Vladimir (1985): Morfología del cuento. Madrid, Akal.

RODRIGO, Miquel (2000): Identitats i comunicació intercultural. Barcelona, Edicions 3 i 4.

TURPIN, Jennifer (1995): Reinventing the Soviet Self: Media and Social Change in the Former Soviet Union. EUA, Praeger, Wesport.

VAN DIJK, Teun A. (1997): Racismo y análisis crítico de los medios. Barcelona, Paidós.

ZASSOURSKY, Ivan (2004): Media and Power in Post-Soviet Russia. New York M.E. Armonk, Sharpe.

ZUNZUNEGUI, Santos (2003): Metamorfosis de la mirada. Museo y semiótica. Madrid, Cátedra. 